\title{
Cut-Open Oocyte Voltage-Clamp Technique
}

Antonios Pantazis and Riccardo Olcese

\section{Book Chapter}

Cite this chapter as:

Pantazis, A., Olcese, R. Cut-Open Oocyte Voltage-Clamp Technique, In Roberts, G., Watts, A., Biophysical, E. (eds), Encyclopedia of Biophysics, Berlin, Heidelberg: Springer Berlin/ Heidelberg; 2019, Living Edition. ISBN: 9783642359439

DOI: https:// doi.org/ 10.1007/ 978-3-642-35943-9 371-1

Copyright: Springer Berlin/Heidelberg

The self-archived postprint version of this journal article is available at Linköping University Institutional Repository (DiVA):

http:// urn.kb.se/ resolve?urn=urn:nbn:se:liu:diva- 162793 


\section{Cut-open Oocyte Voltage Clamp Technique}

Antonios Pantazis ${ }^{1,2,3}$ and Riccardo Olcese ${ }^{1,4,5,6, *}$

${ }^{1}$ Division of Molecular Medicine, Department of Anesthesiology \& Perioperative Medicine, University of California at Los Angeles, Los Angeles,CA, USA

${ }^{2}$ Division of Neurobiology, Department of Clinical and Experimental Medicine (IKE), Linköping University, Linköping, Sweden

${ }^{3}$ Wallenberg Center for Molecular Medicine, Linköping University, Linköping, Sweden

${ }^{4}$ Department of Physiology, David Geffen School of Medicine, University of California at Los Angeles, Los Angeles, CA, USA

${ }^{5}$ Brain Research Institute, University of California at Los Angeles, Los Angeles, CA, USA

${ }^{6}$ Cardiovascular Research Laboratories, David Geffen School of Medicine, University of California at Los Angeles, Los Angeles, CA, USA

${ }^{*}$ Correspondence to:

\section{Riccardo Olcese, Ph.D.}

Professor of Anesthesiology and Physiology

Division of Molecular Medicine, BH 570 CHS

Department of Anesthesiology \& Perioperative Medicine

University of California Los Angeles

Phone: (310) 2062214

Fax: (310) 2061947

Email: rolcese@ucla.edu 


\section{Introduction}

The cut-open oocyte Vaseline gap (COVG) voltage clamp technique, a relatively recent addition to the electrophysiologist's armamentarium, was specifically developed by Drs. Stefani and Bezanilla (Bezanilla et al., 1991) to achieve low-noise recordings of the membrane of Xenopus laevis oocytes with fast clamp speed and thus optimize the most popular transient expression system to reveal the activity voltage-dependent proteins previously difficult to resolve by alternative methods. The high degree of specialization of this technique is complemented by its flexibility: in addition to oocyte perfusion, COVG can be combined with optical measurements (voltage clamp fluorometry and spectroscopy) and flash photolysis for the instantaneous release of intracellular caged compounds, expanding its use beyond electrophysiology.

\section{Historical Points}

During the cloning era, the oocytes of the African clawed frog (Xenopus laevis) emerged as a powerful biological tool, as their large size $(\approx 1 \mathrm{~mm}$ diameter) allowed the micro-injection of cloned RNA into their cytosol, which they readily translated into functional proteins. A class of proteins that particularly benefitted from this expression system are ion channels and transporters, which can be abundantly expressed in the oocyte membrane, particularly with the use of specialized vectors optimized for the Xenopus translation machinery (Cha and Bezanilla, 1997; Shih et al., 1998).

The large size of the oocyte and robust membrane integrity were critical factors in the development of one of the most widely-used electrophysiological techniques: the two-electrode voltage clamp (TEVC). Briefly, TEVC involves impaling the oocyte with two intracellular electrodes; one which measures intracellular potential and another that injects current, so that the measured membrane potential matches the command potential. However, the large size of Xenopus oocytes is a double-edged sword: their physical robustness is countered by their large membrane capacitance, which significantly decreases the voltage clamp speed. Furthermore, 
uniform space clamp (isopotentiality) is difficult to achieve. These drawbacks can be rectified by clamping a much smaller portion of the oocyte membrane (a few $\mu \mathrm{m}^{2}$ ), as achieved by attachedor excised-patch voltage-clamp techniques. However, these techniques can be subject to various drawbacks as well, including low patch stability-especially in macro-patches and the progressive decrease of ion channel activity ('run-down'), usually attributed to the loss of intracellular factors by dialysis or excision, etc.

The drawbacks of TEVC and patch-clamp techniques made it difficult to observe small or transient voltage-dependent charge movements. Specific challenges were $\mathrm{Na}^{+}$channel ionic currents, which inactivate within a few milliseconds, and the 'gating currents', caused by the transmembrane movement of charged residues in voltage-dependent proteins during activation: relatively to ionic currents, they are small and short-lived. The cut-open oocyte Vaseline gap (COVG) voltage clamp technique, the origins of which can be traced to previous Vaseline gap techniques for voltage-clamping cut-open muscle fibres, was specifically developed to maximize the benefits of the Xenopus oocyte expression system, by improving on clamp speed, signal-tonoise ratio and ability to effectively perfuse the oocyte (Taglialatela et al., 1992; Stefani and Bezanilla, 1998). In this way, it was possible to combine the most popular transient expression system, and the associated benefits of molecular cloning and site-directed mutagenesis, with the superior voltage clamp properties of cut-open cell techniques.

\section{Basic Implementation}

Xenopus oocytes are isolated from the ovary by a combination of enzymatic digestion and mechanical perturbation. Their follicular layer (a layer of cells that surround and nourish the developing oocyte) is also removed, either mechanically or enzymatically, as its presence prevents good electrical isolation at the Vaseline gaps of the COVG apparatus (see below). The 
oocytes are then microinjected with the cloned RNA of the gene desired, typically 1-50 ng. After incubation for $2-7$ days at $18^{\circ} \mathrm{C}$, the oocytes express the protein of interest and are ready for voltage clamp.

Briefly, the most basic implementation of COVG involves mounting the oocyte in an apparatus comprising of three electrically-isolated chambers, or pools of solutions, separated by Vaseline gaps. The voltage clamp circuit is then assembled around the mounted oocyte by the placement of six salt bridges and one intracellular electrode, imposing three simultaneous voltage clamps. This is further described below and a schematic representation of the COVG set-up is shown in Fig. 1.
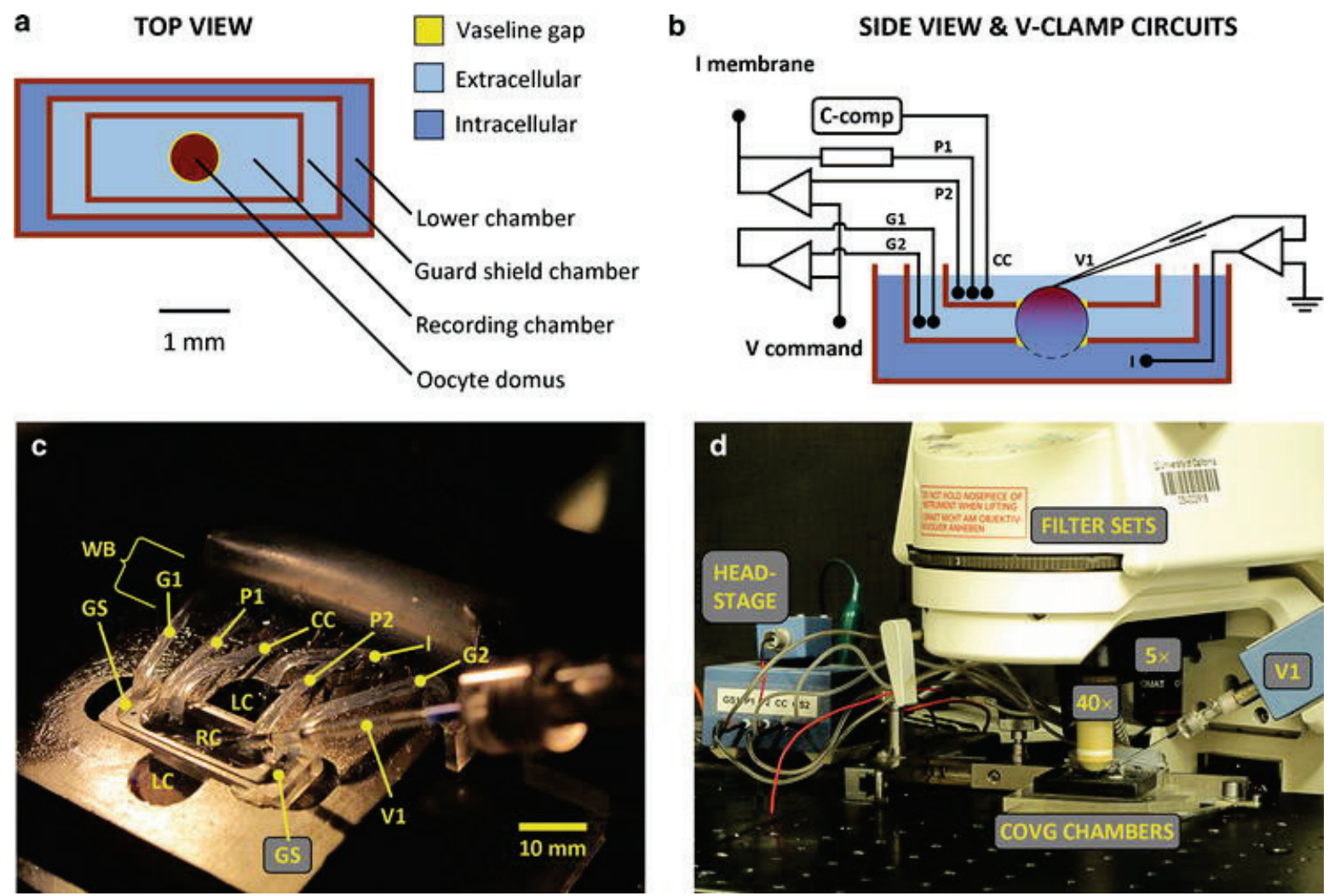

Fig. 1: Schematic representation of the COVG set-up and practical implementation. (A) Top view of the COVG chambers. (B) Side view (cross-section) of the COVG chambers, showing the relative position of the oocyte, the Vaseline gaps (in yellow) and a simplified diagram of the circuitry. The oocyte membrane immersed in the lower chamber (dashed line) has been permeabilized with $0.1 \%$ saponin, to electrically connect the ooplasm with the current-injecting 
bridge I. (C) Photograph of the COVG chambers. WB: well block; LC: lower chamber; GS: guard shield chamber; RC: recording chamber; V1: intracellular electrode; G1, G2, P1, P2, CC: Salt bridges as shown in panel B. (D) The same COVG chambers in a set-up optimized for voltageclamp fluorometry (see Fig. 3) using a Zeiss Axiovert FS epifluorescence microscope. A 40x objective is immersed in the RC solution to transmit excitation light and collect fluorescence emission.

The three chambers or the COVG are referred to, respectively from top to bottom, as recording, guard shield, and lower chamber. The recording and guard chambers possess $0.6 \mathrm{~mm}$ holes to accommodate the oocyte. The holes are lined with Vaseline ${ }^{\circledR}$ (or a viscous mixture of mineral oil and molten Parafilm ${ }^{\circledR}$ ) so that when the oocyte is mounted, the chambers are electrically isolated; thus the Vaseline gaps are formed. A commercially-available electrophysiological amplifier for COVG is the Dagan CA-1B (Dagan Corporation, MN, USA). The electronic circuitry of the amplifier terminates in $\mathrm{Ag} / \mathrm{AgCl}$ electrode coils lining wells filled with $1 \mathrm{M}$ $\mathrm{NaCl}$. The wells are connected to the chambers via 1-2 cm agar (3\%) bridges typically containing $1 \mathrm{M} \mathrm{Na}$ methanesulfonate, threaded up to $\approx 0.5 \mathrm{~mm}$ from their ends with $\mathrm{Pt}$ wire to improve admittance.

Mounting the oocyte begins by resting it on the hole separating the lower and guard chambers. Aspirating some of the LC solution will develop positive hydrostatic pressure in the GS chamber, which will hold the oocyte in place. Then, the top chamber can be placed over the oocyte, so that its upper dome $(\approx 20 \%$ of the total oocyte surface $)$ is electrically isolated from the guard chamber below: a manoeuvre that can be facilitated by using a stereoscope. The oocyte's lower part is exposed to the lower chamber, and can be cut or permeabilized by brief (up to 1 min) exposure to $0.1 \%$ saponin, so that the ooplasm becomes electrically continuous with the internal solution pool in the lower chamber. Thus, the lower chamber contains "internal solution" 
of typically high $\left[\mathrm{K}^{+}\right]$composition, while he guard and recording chambers contain "external solution" with higher $\left[\mathrm{Na}^{+}\right]$, according to the experimental protocol. Two voltage clamps are used to simultaneously clamp the guard and recording chamber solutions to the opposite value of the command potential, each requiring the placement of two salt bridges. A third voltage clamp injects current intracellularly through the lower chamber to actively ground the ooplasm beneath the domus membrane, as measured by a sharp electrode impaling the oocyte domus in the recording chamber. In this way, the membrane potential is clamped to the command potential. Optimal space clamp is achieved when the voltage electrode has impaled the oocyte apex (Perozo et al., 1992). During placement, the electrode is easier to see against a dark background, achieved by mounting the oocyte with the animal pole facing up. As in other voltage-clamp systems, circuitry allows for series resistance compensation, leak subtraction and capacitative transient compensation — the latter using an additional bridge in the recording chamber

\section{COVG Advantages and Scientific Breakthroughs}

Even at its most basic implementation described above, COVG is a more complex technique than TEVC or patch clamp. However, COVG is capable of fast voltage steps, with a clamp speed in the order of $20-40 \mu$ s and relatively low noise $(\approx 1 \mathrm{nA}$ r.m.s. at $5 \mathrm{kHz})$. These advantages can be attributed to the following specific features:

1. Low access resistance for intracellular current injection due to the physical ablation or chemical permeabilization of a large portion of the oocyte membrane at the lower chamber.

2. The electrical isolation of the voltage-clamped guard and recording chambers helps to achieve isopotentiality at the recording chamber.

In this respect, COVG possesses better voltage clamp properties than TEVC, applied with more stability, less run-down and over a much larger area of the oocyte than patch techniques. In addition, COVG allows extensive control of the perfusing solutions, further discussed below. 
These benefits resulted in the resolution and characterization of extremely small and transient voltage-dependent transmembrane charge movements that allowed the investigation of voltage-dependent channel gating, in particular the gating currents from Shaker (Bezanilla et al., 1991) and DRK1 potassium channels (Taglialatela and Stefani, 1993); Voltage gated sodium and calcium channels (Neely et al., 1993; Cha et al., 1999); EAG (Tang et al., 2000) and HERG potassium channels (Piper et al., 2003); large-conductance voltage- and $\mathrm{Ca}^{2+}$-activated $\mathrm{K}^{+}(\mathrm{BKCa}$ or MaxiK) channels (Savalli et al., 2006) and Kv7 channels (Miceli et al., 2009).

In addition, COVG was used to reveal the 'gating currents' of an ascidian phosphoinositide phosphatase (Ci-VSP) (Murata et al., 2005) as well as muscarinic ACh receptors (Ben Chaim et al., 2006), directly demonstrating the intrinsic voltage dependence of these proteins. Representative gating current traces from $\mathrm{BK}_{\mathrm{Ca}}$ and Shaker $\mathrm{K}^{+}$channels are shown in Fig. 2.

a

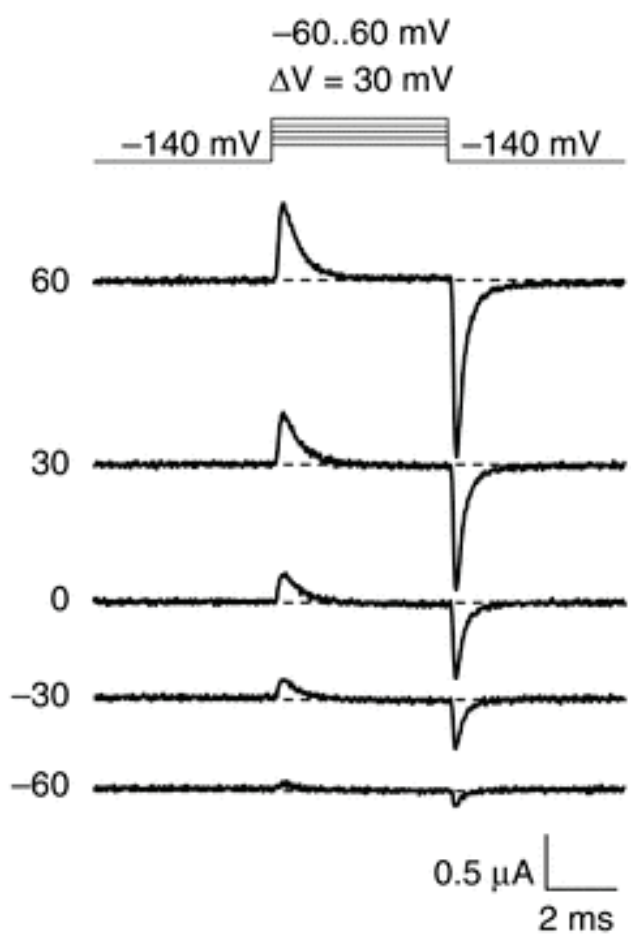

b Shaker channels

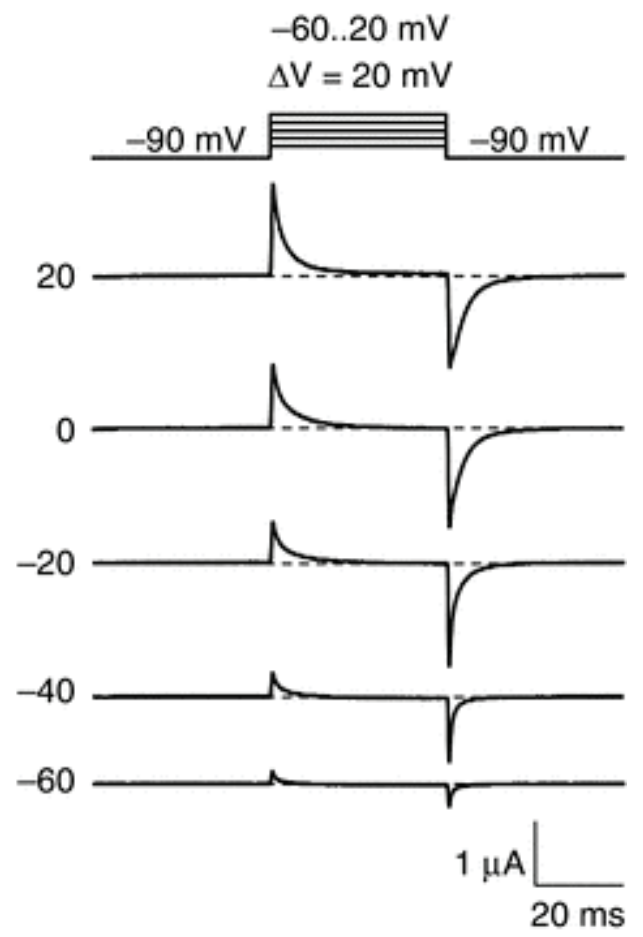

Fig. 2: Gating currents from $B K c_{a}$ and Shaker $\mathrm{K}^{+}$channels. (A) A family of gating currents recorded using COVG from an oocyte expressing BKca (or MaxiK) channels (hSlo(M4)-C14SR20A-C141S-S202C-W203V-R207Q-C277S). Zero current is indicated by the dashed line. The 
oocyte was voltage-clamped using the protocol shown above the traces. The test potential (in $\mathrm{mV}$ ) is shown on the left of each trace. (B) As in panel A, for Shaker $\mathrm{K}^{+}$channels (Shaker $\mathrm{H} 4-(\Delta 6-$ 46)-M356C-W434F). Note that Shaker channels have slower gating kinetics.

\section{Solution Manipulation in COVG}

In some experiments, the ability to manipulate the perfusing solutions can be as important as fast voltage control. Since only a defined portion of the oocyte membrane is clamped in COVG (the domus exposed in the recording chamber), the external solution is easier to manipulate than in the TEVC technique due to the large surface area and spherical geometry of the oocyte. The recording chamber solution is easily accessible and can be aspirated almost entirely before exchange during the experiment. Alternatively, concentrated stocks of drugs or modifying agents can be manually added into the existing external solution. More controlled solution exchange can be implemented with a Picospritzer.

The internal solution is harder to control in the basic COVG implementation as described above due to diffusion barriers in the ooplasm. It is possible to micro-inject substances prior to the experiment; indeed, injecting on the portion of the oocyte to be permeabilzed at the lower chamber ensures that the injection will not cause membrane leak. During the experiment, a perfusion cannula or pipette can be introduced intracellularly through the lower chamber (Taglialatela et al., 1992; Stefani and Bezanilla, 1998). One use of this is the fast and complete depletion of intracellular conducting ions, as in the investigation of gating currents from unblocked, wild-type Shaker $\mathrm{K}^{+}$channels (Olcese et al., 1997). Despite these manoeuvres, run-down remains minimal (Stefani and Bezanilla, 1998). Furthermore, molecules chelated by UV-sensitive molecular cages $\left(\mathrm{Ca}^{2+}\right.$, protons, etc.) can be micro-injected prior to the experiment. The caged compounds can then be instantaneously released by flash photolysis, with UV light directed at the oocyte domus using a light guide. 
Temperature jumps can be executed by perfusing with external solution of the desired temperature. Stable temperature control can be achieved by including thermoelectric (Peltier) elements in the set-up, while a thermistor probe in a negative feedback circuit with the Peltier element can provide automated temperature control (Stefani and Bezanilla, 1998).

\section{Combination of the Cut-open Oocyte Voltage Clamp with Optical Techniques}

Voltage clamp fluorometry (VCF) is a new, innovative approach for optically tracking protein conformational rearrangements. It is discussed in depth elsewhere in this book. In this section, VCF will be briefly introduced in the context of a COVG set-up. The central principle of VCF is that many fluorophores are sensitive to their local environment (Lakowicz, 2006). By engineering such fluorophores with a sulfydryl-reactive moiety, such as maleimide, it is possible to conjugate them to a specific protein site where a unique, accessible cysteine has been introduced. If this area of the protein then undergoes a motion that results in a change of the fluorophore environment, a deflection in the fluorescence intensity will be observed. VCF was pioneered in Shaker $\mathrm{K}^{+}$channels expressed in oocytes clamped by TEVC (Mannuzzu et al., 1996), but was shortly after implemented in COVG (Cha and Bezanilla, 1997). Since then, COVG and VCF have been combined (COVG-F) to resolve voltage-dependent conformational rearrangements in many other channels and voltage-sensitive proteins (Cha et al., 1999; Smith and Yellen, 2002; Bannister et al., 2005; Savalli et al., 2006; Villalba-Galea et al., 2008, Pantazis et al., 2014).

The practical implementation of COVG-F is extensively described in (Gandhi and Olcese, 2008). The set-up is illustrated in Fig. 3. Briefly, oocytes expressing ion channels with a single extracellular cysteine are incubated with the maleimide fluorophore to allow conjugation. The optimal conditions for the fluorescence labelling of proteins vary according to fluorophore reactivity and the accessibility of the cysteine. A labeling technique for BKca channels with tetramethylrhodamine-5'-maleimide (TMRM) is to incubate the oocytes in depolarizing solution 
(120 mM K methanesulfonate, $10 \mathrm{mM} \mathrm{HEPES,} \mathrm{pH}=7.0$ ) with $10 \mu \mathrm{M}$ TMRM, at room temperature, for 30-45' in the dark (Savalli et al., 2006; Savalli et al., 2007; Pantazis et al., 2010a; Pantazis et al., 2010b). After thorough washing in dye-free solution, the oocyte is mounted on a COVG setup which has been modified for epifluorescence measurement as shown in Fig. 3, while Fig. 4 shows simultaneously-recorded ionic currents and fluorescence signals from TMRM-labeled BKca and Shaker $\mathrm{K}^{+}$channels. 
a
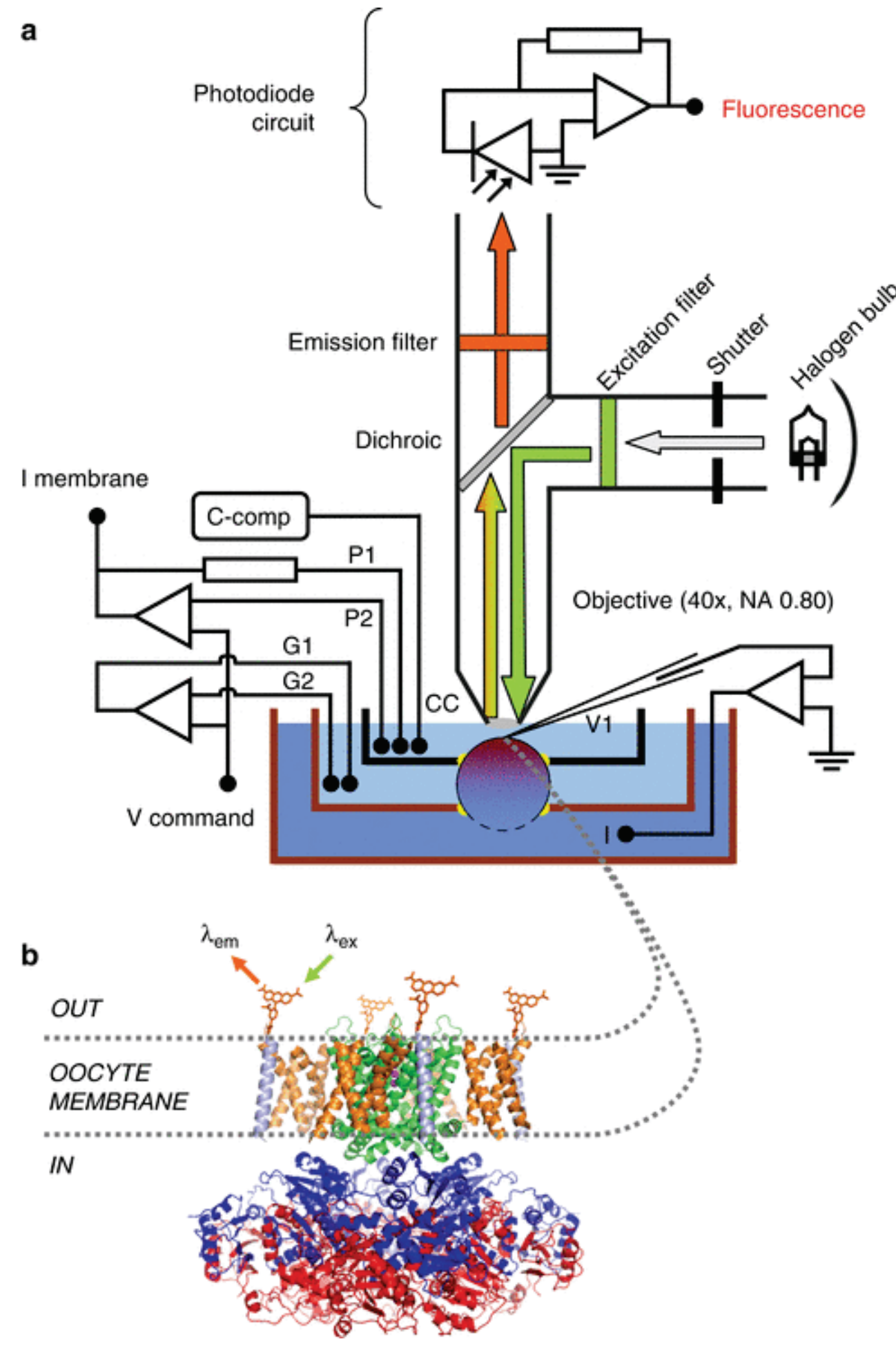

Fig. 3: COVG technique combined with fluorometry. (A) The COVG chambers (Fig. 1) in an epifluorescence microscope to simultaneously record membrane current and fluorescence from environment-sensitive fluorophores site-specifically labeling the expressed channels. (B) A BKca channel in the oocyte membrane (putative structure), which have been conjugated with tetramethylrhodamine-5'-maleimide (TMRM) fluorophores. 
a
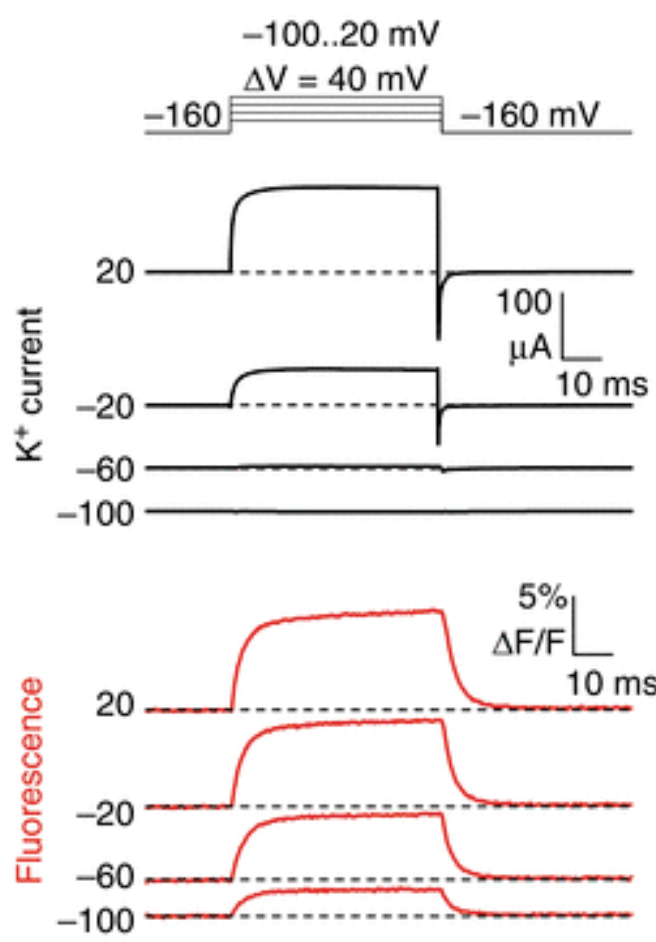

b Shaker channels
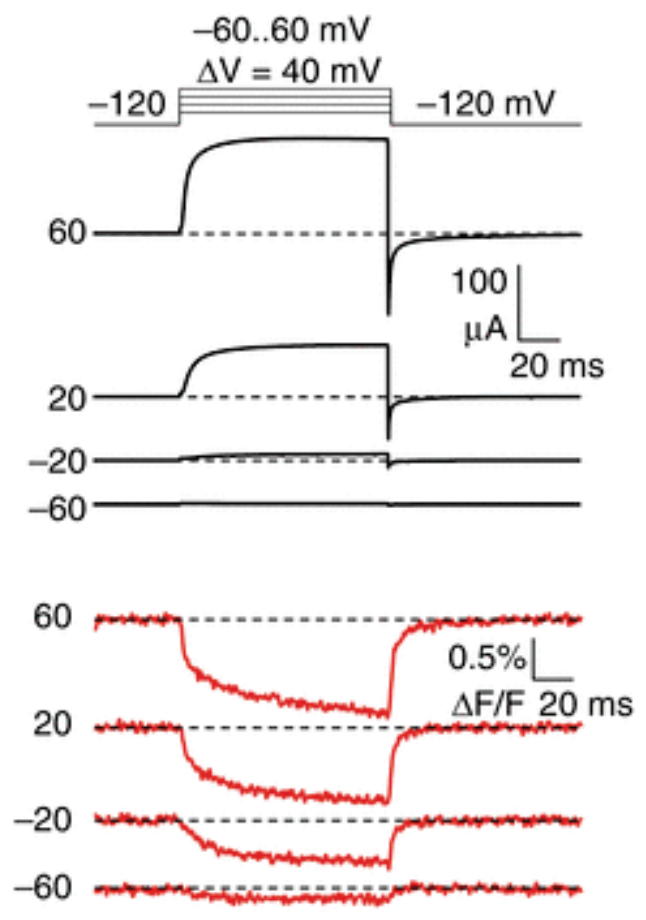

Fig. 4: Simultaneously-resolved ionic currents and fluorescence with COVG. (A) A family of $\mathrm{K}^{+}$currents (black traces) and simultaneously-resolved fluorescence deflections (red traces) from BKca channels labeled with TMRM outside their S0 transmembrane segment ( $h$ Slo(M4)-C14SG18C-C141S-R207Q-C277S). The S0-bound TMRM is quenched by a tryptophan residue outside the S4 segment (W203) in a state-dependent manner. The fluorescence deflections correspond to the relative voltage-dependent motion of the two transmembrane helices (Pantazis et al., 2010b). (B) As in panel A for non-inactivating Shaker $\mathrm{K}^{+}$channels labeled with TMRM outside their S4 segment (Shaker H4-( $\Delta 6-46)-M 356 C)$. The fluorescence deflections are related to motions that the channel undergoes during gating (Mannuzzu et al., 1996; Cha and Bezanilla, 1997). Therefore, even though the observed voltage-dependent $\Delta \mathrm{F}$ exhibit opposite sign and different amplitude and kinetics in the two channels, they likely report functionally related structural changes. 
An upright microscope with a $40 \times$ water-immersion objective lens (N.A. $=0.80)$ is used, so that the entire oocyte domus in the field of view. A halogen bulb $(12 \mathrm{~V}, 100 \mathrm{~W})$ can be used as a light source, connected to a stable power supply to prevent light fluctuations. The light passes through the excitation filter and is reflected by the dichroic, through the objective lens, onto the oocyte domus, at a wavelength that excites the fluorophore. Emitted light passes through the objective, the dichroic and the emission filter, to reach the light detection circuit. Fig. 4 shows characteristic ionic currents and fluorescence deflections from TMRM-labelled BKca and Shaker $\mathrm{K}^{+}$channels, simultaneously resolved using the methods described above. Although a photodiode is used as an example to record fluorescence in Fig. 3, a photomultiplier tube or a charge-coupled camera can be used instead. The emitted light can also be sampled by a spectrometer, as the spectral properties of the emitted light can yield information on the environmental changes experienced by the conjugated fluorophore (Cha and Bezanilla, 1997).

To ensure that only the fluorescent light emitted from ion channels embedded in the voltage-clamped portion of the oocyte is detected, an opaque recording chamber should be used. The ability to detect fluorescence from the entire voltage-clamped area of the oocyte while excluding photons from unclamped regions is a major advantage of combining VCF with COVG over TEVC, in addition to the voltage clamp and solution exchange benefits discussed above.

\section{Summary}

The cut-open oocyte Vaseline gap (COVG) voltage clamp technique was developed during the height of the cloning era, to optimize voltage clamp on the Xenopus oocyte transient expression system. It offered the following advantages to the electrophysiologist:

1. Very fast (20-40 $\mu$ s), uniform voltage clamp of a large $(20 \%)$, defined area of the oocyte. Low noise (1 nA r.m.s. at $5 \mathrm{kHz}$ ) compared with TEVC. Therefore, a great number of expressed proteins can be investigated under optimal voltage clamp conditions. 
2. Ability to exchange both internal and external solutions during the experiment, yet exhibit good stability and minimal run-down for several hours.

3. Versatility for a wide spectrum of applications, including fast intracellular release of caged compounds and voltage-clamp fluorometry. 
Cross-References

$\rightarrow$ Channel Gating

$\rightarrow$ Clamp Speed

$\rightarrow$ Flash photolysis

$\rightarrow$ Fluorescence

$\rightarrow$ Fluorescence labeling of proteins

$\rightarrow$ Fluorescence quenching

$\rightarrow$ Fluorescence based techniques for studying ion channel gating - voltage clamp fluorometry LRET, FRET

$\rightarrow$ Labeling technique

$\rightarrow$ Potassium channels

$\rightarrow$ Space clamp

$\rightarrow$ Structural basis for voltage dependent gating of Kv and Kir channels

$\rightarrow$ Voltage gated sodium and calcium channels 


\section{References}

Bannister, J.P., B. Chanda, F. Bezanilla, and D.M. Papazian. 2005. Optical detection of ratedetermining ion-modulated conformational changes of the ether-a-go-go $\mathrm{K}+$ channel voltage sensor. Proc. Natl. Acad. Sci. U. S. A. 102:18718-18723.

Ben Chaim, Y., B. Chanda, N. Dascal, F. Bezanilla, I. Parnas, and H. Parnas. 2006. Movement of 'gating charge' is coupled to ligand binding in a G-protein-coupled receptor. Nature. 444:106-109.

Bezanilla, F., E. Perozo, D.M. Papazian, and E. Stefani. 1991. Molecular basis of gating charge immobilization in Shaker potassium channels. Science. 254:679-683.

Cha, A. and F. Bezanilla. 1997. Characterizing voltage-dependent conformational changes in the Shaker K+ channel with fluorescence. Neuron. 19:1127-1140.

Cha, A., P.C. Ruben, A.L. George, Jr., E. Fujimoto, and F. Bezanilla. 1999. Voltage sensors in domains III and IV, but not I and II, are immobilized by Na+ channel fast inactivation. Neuron. 22:73-87.

Gandhi, C.S. and R. Olcese. 2008. The Voltage-Clamp Fluorometry Technique. In Methods in Molecular Biology, Potassium Channels. J.D. Lippiat, editor. Humana Press. Totowa. 213-231.

Lakowicz, J.R. 2006. Principles of Fluorescence Spectroscopy. 3rd ed. Springer. New York, NY. $954 \mathrm{pp}$.

Mannuzzu, L.M., M.M. Moronne, and E.Y. Isacoff. 1996. Direct physical measure of conformational rearrangement underlying potassium channel gating. Science. 271:213216. 
Miceli, F., M.R. Cilio, M. Taglialatela, and F. Bezanilla. 2009. Gating currents from neuronal $\mathrm{K}(\mathrm{V}) 7.4$ channels: general features and correlation with the ionic conductance. Channels (Austin. ). 3:274-283.

Murata, Y., H. Iwasaki, M. Sasaki, K. Inaba, and Y. Okamura. 2005. Phosphoinositide phosphatase activity coupled to an intrinsic voltage sensor. Nature. 435:1239-1243.

Neely, A., X. Wei, R. Olcese, L. Birnbaumer, and E. Stefani. 1993. Potentiation by the beta subunit of the ratio of the ionic current to the charge movement in the cardiac calcium channel. Science. 262:575-578.

Olcese, R., R. Latorre, L. Toro, F. Bezanilla, and E. Stefani. 1997. Correlation between charge movement and ionic current during slow inactivation in Shaker K+ channels. J. Gen. Physiol. 110:579-589.

Pantazis, A., V. Gudzenko, N. Savalli, D. Sigg, and R. Olcese. 2010a. Operation of the voltage sensor of a human voltage- and Ca2+-activated K+ channel. Proc. Natl. Acad. Sci. U. S. A. 107:4459-4464.

Pantazis, A., A.P. Kohanteb, and R. Olcese. 2010b. Relative motion of transmembrane segments $\mathrm{SO}$ and $\mathrm{S} 4$ during voltage sensor activation in the human $\mathrm{BK}(\mathrm{Ca})$ channel. $J$. Gen. Physiol. 136:645-657.

Pantazis, A., N. Savalli, D. Sigg, A. Neely, and R. Olcese. 2014. Functional heterogeneity of the four voltage sensors of a human L-type calcium channel. Proc. Natl. Acad. Sci. U. S. A. 111:18381-18386.

Perozo, E., D.M. Papazian, E. Stefani, and F. Bezanilla. 1992. Gating currents in Shaker K+ channels. Implications for activation and inactivation models. Biophys. J. 62:160-168. 
Piper, D.R., A. Varghese, M.C. Sanguinetti, and M. Tristani-Firouzi. 2003. Gating currents associated with intramembrane charge displacement in HERG potassium channels. Proc. Natl. Acad. Sci. U. S. A. 100:10534-10539.

Savalli, N., A. Kondratiev, S.B. de Quintana, L. Toro, and R. Olcese. 2007. Modes of operation of the BKCa channel beta2 subunit. J. Gen. Physiol. 130:117-131.

Savalli, N., A. Kondratiev, L. Toro, and R. Olcese. 2006. Voltage-dependent conformational changes in human $\mathrm{Ca}(2+)$ - and voltage-activated $\mathrm{K}(+)$ channel, revealed by voltageclamp fluorometry. Proc. Natl. Acad. Sci. U. S. A. 103:12619-12624.

Shih, T.M., R.D. Smith, L. Toro, and A.L. Goldin. 1998. High-level expression and detection of ion channels in Xenopus oocytes. Methods Enzymol. 293:529-556.

Smith, P.L. and G. Yellen. 2002. Fast and slow voltage sensor movements in HERG potassium channels. J. Gen. Physiol. 119:275-293.

Stefani, E. and F. Bezanilla. 1998. Cut-open oocyte voltage-clamp technique. Methods Enzymol. 293:300-318.

Taglialatela, M. and E. Stefani. 1993. Gating currents of the cloned delayed-rectifier K+ channel DRK1. Proc. Natl. Acad. Sci. U. S. A. 90:4758-4762.

Taglialatela, M., L. Toro, and E. Stefani. 1992. Novel voltage clamp to record small, fast currents from ion channels expressed in Xenopus oocytes. Biophys. J. 61:78-82.

Tang, C.Y., F. Bezanilla, and D.M. Papazian. 2000. Extracellular Mg(2+) modulates slow gating transitions and the opening of Drosophila ether-a-Go-Go potassium channels. J. Gen. Physiol. 115:319-338. 
Villalba-Galea, C.A., W. Sandtner, D.M. Starace, and F. Bezanilla. 2008. S4-based voltage sensors have three major conformations. Proc. Natl. Acad. Sci. U. S. A. 105:1760017607. 